\title{
Acute appendicitis: transcript profiling of blood identifies promising biomarkers and potential underlying processes
}

\author{
Lakhmir S. Chawla ${ }^{1,6}$, lan Toma², Danielle Davison" ${ }^{1}$, Khashayar Vaziri ${ }^{3}$, Juliet Lee ${ }^{1,3}$, Raymond Lucas ${ }^{5}$, \\ Michael G. Seneff ${ }^{5}$, Aoibhinn Nyhan ${ }^{2}$ and Timothy A. McCaffrey ${ }^{2,4^{*}}$
}

\begin{abstract}
Background: The diagnosis of acute appendicitis can be surprisingly difficult without computed tomography, which carries significant radiation exposure. Circulating blood cells may carry informative changes in their RNA expression profile that would signal internal infection or inflammation of the appendix.

Methods: Genome-wide expression profiling was applied to whole blood RNA of acute appendicitis patients versus patients with other abdominal disorders, in order to identify biomarkers of appendicitis. From a large cohort of emergency patients, a discovery set of patients with surgically confirmed appendicitis, or abdominal pain from other causes, was identified. RNA from whole blood was profiled by microarrays, and RNA levels were filtered by a combined fold-change $(>2)$ and $p$ value $(<0.05)$. A separate set of patients, including patients with respiratory infections, was used to validate a partial least squares discriminant (PLSD) prediction model.

Results: Transcript profiling identified 37 differentially expressed genes (DEG) in appendicitis versus abdominal pain patients. The DEG list contained 3 major ontologies: infection-related, inflammation-related, and ribosomal processing. Appendicitis patients had lower level of neutrophil defensin mRNA (DEFA1,3), but higher levels of alkaline phosphatase (ALPL) and interleukin-8 receptor-B (CXCR2/IL8RB), which was confirmed in a larger cohort of 60 patients using droplet digital PCR (ddPCR).

Conclusions: Patients with acute appendicitis have detectable changes in the mRNA expression levels of factors related to neutrophil innate defense systems. The low defensin mRNA levels suggest that appendicitis patient's immune cells are not directly activated by pathogens, but are primed by diffusible factors in the microenvironment of the infection. The detected biomarkers are consistent with prior evidence that biofilm-forming bacteria in the appendix may be an important factor in appendicitis.
\end{abstract}

Keywords: Appendicitis, Transcript profiling, Biomarkers, Interleukin-8 receptor, Alkaline phosphatase, Defensin

\section{Background}

Abdominal pain is a major cause of hospital visits, accounting for about $10 \%$ of 62 million visits per year by adults who present at an emergency department (ED) for non-injury causes [1]. Acute appendicitis is one of

\footnotetext{
*Correspondence: mcc@gwu.edu

${ }^{2}$ Department of Medicine, Division of Genomic Medicine, The George Washington University Medical Center, 2300 I Street, NW Ross 443, Washington, DC 20037, USA

${ }^{4}$ Department of Microbiology, Immunology, and Tropical Medicine, The George Washington University Medical Center, 2300 I Street, NW Ross 443, Washington, DC 20037, USA

Full list of author information is available at the end of the article
}

the most common causes of abdominal pain and results in nearly 750,000 ED visits with approximately 250,000 appendectomies performed annually. Globally, a small but significant portion of the operations are "negative appendectomies", resulting in the removal of a noninflamed appendix due to misdiagnosis [2-4], reported as high as 17-28 \% outside the United States (US) and Western Europe [5, 6].

Prior to the widespread availability of computed tomography $(\mathrm{CT})$ scans, the accurate diagnosis of appendicitis could be challenging, and in places where $\mathrm{CT}$ is still not available, the Alvarado score of clinical characteristics is a 
widely used diagnostic tool $[5,6]$. Currently in the US, CT scanning is the 'gold standard' for the diagnosis of appendicitis, with magnetic resonance imaging (MRI) being a reasonable alternative in pregnant women [7], and ultrasound sonography being an acceptable alternative for preliminary diagnostics to avoid radiation [8]. While CT is the most sensitive and specific diagnostic tool for appendicitis $[9,10]$, and used in almost $98 \%$ of patients undergoing appendectomy in the US [11], CT scanning carries a significant radiation exposure, and epidemiologic data suggest that radiation exposure can increase the risk of developing a future malignancy [12]. This issue is of particular concern in children because they are more sensitive to the hazards of radiation, they are among the most common patients to present to the ED with abdominal pain, and have the highest rate of misdiagnosis [10, 13]. In an attempt to reduce the damaging effect of CT scans, several clinical trials are examining the diagnostic utility of lower doses of radiation, primarily in children [14-16].

In order to understand the landscape of the immune reaction to appendicitis, we tested the hypothesis that microarray profiling of whole blood RNA would identify blood biomarkers of appendicitis. Toward that goal, we employed genome-wide profiling of RNA transcripts in whole blood RNA of patients presenting at the ED for abdominal pain, resulting in confirmed appendicitis versus other abdominal abnormalities. Ultimately, there is the potential to use blood RNA biomarkers as a prescreening method to utilize CT scanning more appropriately, and to improve diagnosis in areas where CT scans are unavailable.

\section{Methods}

\section{Subjects}

\section{Ethics statement}

The protocol of this observational study was approved by the Institutional Review Board of The George Washington University, and all subjects gave informed consent. From a cohort of 270 patients presenting to the ED for various reasons, a subset of 40 subjects with a principal complaint of abdominal pain, and who met inclusion/exclusion criteria (Additional file 1: Table S1), were identified, and divided into a discovery set of 20 patients, and a validation set of 20 patients for transcript profiling of whole blood RNA by microarray.

Discovery Set For the discovery set, we employed 20 subjects who presented to the ED who were undergoing CT scanning. In order to meet criteria, the patient undergoing the CT scan must have had appendicitis suspected in the differential diagnosis. Appendicitis Patients: Patients with appendicitis were diagnosed by CT scanning $(n=11)$, and had research blood samples drawn by venipuncture after anesthetic induction, but prior to skin incision for appendectomy. All cases of appendicitis were confirmed by surgical inspection and histopathology, including mucosal ulceration and neutrophil infiltration of the lamina propria and/or perivascular space. Control Patients: Patients included in the control arm $(n=9)$ were patients who were found not to have appendicitis, by both $\mathrm{CT}$ scanning and clinical follow-up. This included patients with reported abdominal pain (ABD), later found to be caused by diverticulitis, colitis, or other gastrointestinal pathologies, but not clinically associated with appendicitis. Blood was drawn at study enrollment for these patients.

Validation Set: control patients Because appendicitis can involve infection, 5 patients with lower respiratory tract infections (LRI) in the ED were enrolled as an 'infection' control. Also, as a control for surgical factors, 5 patients undergoing elective ventral hernia or inguinal hernia repair (HER) were enrolled, and they were compared with 9 new patients with surgically confirmed appendicitis (APP). In all surgical patients, including appendicitis and hernia repairs, research blood samples were drawn by venipuncture after anesthetic induction, and prior to skin incision. Two patients, (1 HER, 1 APP) were excluded due to technical complications in RNA purification or microarray analysis. Clinical diagnoses on all patients analyzed are available in Additional file 2: Table S2.

\section{Blood samples}

Blood was drawn in $3.2 \%$ sodium citrate tubes for frozen plasma samples, in Tempus Blood RNA tubes (ABI) for genome-wide RNA profiling, and in BD Vacutainer K2 tubes for complete blood counts with differentials.

\section{RNA purification for transcript profiling}

Tempus Blood RNA preservation tubes were stored at $-80^{\circ} \mathrm{C}$ and then thawed at $37^{\circ} \mathrm{C}$ prior to processing according to manufacturer's methods. Total RNA was purified from whole blood using Tempus Blood RNA kit (ABI), followed by an aggressive DNAse treatment. Briefly, the preserved whole blood was pelleted at $3000 \mathrm{x}$ $\mathrm{g}$ for $30 \mathrm{~min}$ in a $4{ }^{\circ} \mathrm{C}$ refrigerated centrifuge, redissolved in lysis buffer, and nucleic acids were bound to a column. After washing, nucleic acids were eluted with nuclease-free water and quantified with a NanoDrop ND-1000 spectrophotometer. DNA was eliminated by aggressive DNAse treatment (TurboDNAse, Ambion) at $2 \mathrm{U} / 10 \mu \mathrm{g}$ nucleic acids, followed by affinity removal of the DNAse. The remaining RNA was quantified by 260/ 280 ratio by NanoDrop, and RNA integrity was evaluated by capillary electrophoresis on a Bioanalyzer 2100 (Agilent). RIN scores $>7$ were considered acceptable for further sample processing and did not differ between groups. 


\section{Microarray expression profiling and analysis}

Purified RNA (100 ng) was labeled with the Illumina cRNA synthesis kit and hybridized to Illumina Human HT-12v4 Expression BeadChip arrays containing 45,966 probes derived from the NCBI RefSeq release 38. The arrays were washed and then fluorescence was quantitated on an Illumina HiScan.

The fluorescence levels per bead were converted to transcript levels using Illumina GeneStudio, which averaged $\sim 30$ beads per transcript to produce a mean expression level for each of the $46 \mathrm{~K}$ transcripts. Raw BeadChip fluorescence values were imported into GeneSpring GX12.5 with normalization to the $75^{-}$percentile of expression, but without baseline transformation. The main effect of identifying differentially expressed genes (DEG) with respect to appendicitis versus controls was achieved by a combined filter for a $p$ value $<0.05$ on $t$ test without correction for multiple testing, and 2) fold change $>2.0$. The DEG list was further analyzed for gene ontologies using DAVID [17]. Using the DEG list, a partial least squares discriminant (PLSD) prediction model was built in GeneSpring and internally validated with a Leave One Out Cross Validation (LOOCV) algorithm. The PLSD model was externally tested by applying the algorithm to a separate validation set of microarray samples not involved in building the model.

\section{Validation by droplet digital PCR (ddPCR)}

In addition to the patient samples used for the discovery and validation microarray studies, an additional 29 patients (12 APP, $17 \mathrm{ABD}$ ) were drawn from the same cohort, to compose a cohort for ddPCR validation (30 APP, $25 \mathrm{ABD}$, 5 HER). RNA was purified from Tempus-preserved whole blood as described above and DNAse-treated prior to quantitative PCR using a ddPCR system (BioRad QX200). Total RNA was reverse transcribed with an RNAseH+ reverse transcriptase (iScript, BioRad) using random hexamer primers. The cDNA was purified and amplified with transcript-specific primers for ALPL, IL8RB, DEFA1, and ACTB (Additional file 3: Table S3). ddPCR uses clear oil to create thousands of individual nanoliter-sized droplets containing CDNA and PCR reagents with a fluorescent detection dye EVAgreen. The number of positive droplets is proportional to the abundance of the cDNA target of interest, and can be calculated in absolute quantity from a Poisson distribution. To account for variations between RNA preparations, the quantity of target transcripts ALPL, IL8RB, and DEFA1 was expressed as a \% of the ACTB levels in the sample.

\section{Statistical analysis}

Summary statistics are reported as mean and standard error of measurement (SEM). Comparisons between groups are made by an unpaired Student's $t$ test with correction for multiple testing using the method of Benjamini and Hochberg, unless otherwise specified. Correlations between multiple measures of samples were computed as the Pearson r statistic.

\section{Results}

\section{Clinical parameters}

As shown in Table 1, the clinical parameters between patients presenting with appendicitis versus other abdominal indications in the discovery set were generally similar. Age, gender, and body mass index (BMI) were comparable, although the appendicitis patients in the discovery set were all Caucasian. Notably, white blood cell (WBC) counts were comparable, but appendicitis

Table 1 Demographic, clinical, and laboratory findings of patients in the discovery cohort

\begin{tabular}{|c|c|c|c|c|}
\hline & & Unit & APP (11) & $\mathrm{ABD}(9)$ \\
\hline Gender & & $\% \mathrm{M}$ & 54.55 & 55.56 \\
\hline \multirow[t]{2}{*}{ Age } & Mean & Years & 40.73 & 45.89 \\
\hline & SD & & 15.45 & 15.54 \\
\hline \multirow[t]{2}{*}{ BMl } & Mean & $\mathrm{BMI}$ & 24.51 & 26.44 \\
\hline & SD & & 4.92 & 4.48 \\
\hline \multirow[t]{2}{*}{ Race } & & $\% C$ & 100.00 & 55.56 \\
\hline & & $\% A A$ & 0.00 & 44.44 \\
\hline Smoker & & $\%$ & 18.18 & 11.11 \\
\hline \multirow[t]{2}{*}{ Duration of Symptom } & Mean & Hours & 29.45 & 32.75 \\
\hline & SD & & 18.68 & 30.65 \\
\hline \multirow[t]{2}{*}{ Temperature } & Mean & Celsius & 36.97 & 36.80 \\
\hline & SD & & 0.47 & 0.38 \\
\hline \multirow[t]{2}{*}{ WBC } & Mean & K/ul & 13.06 & 13.23 \\
\hline & SD & & 6.44 & 30.65 \\
\hline Elevated Neutrophils & $>75 \%$ & $\%$ & 55.00 & 37.50 \\
\hline \multirow[t]{2}{*}{ Neutrophils } & Mean & $\% W B C$ & 77.18 & 70.00 \\
\hline & SD & & 8.76 & 10.14 \\
\hline \multirow[t]{2}{*}{ Creatinine } & Mean & & 0.78 & $1.54^{*}$ \\
\hline & SD & & 0.13 & 1.06 \\
\hline $\mathrm{pH}$ & $<7.35$ & $\%$ & 0.00 & 11.11 \\
\hline $\mathrm{Na}<130$ & & $\%$ & 0.00 & 0.00 \\
\hline $\mathrm{HCT}<30$ & & $\%$ & 0.00 & 11.11 \\
\hline Glu $>250$ & & $\%$ & 0 & 0 \\
\hline Total RNA conc. & Mean & $\mathrm{ng} / \mathrm{ul}$ & 102.36 & 66.48 \\
\hline Immunosupressed & SD & & 72.49 & 34.06 \\
\hline Folds amp. & Mean & fold & 67.96 & 64.13 \\
\hline Antibiotic use & SD & & 60.48 & 35.81 \\
\hline \multirow[t]{2}{*}{ Defensin Score } & Mean & RNA level & 1.26 & $2.62^{*}$ \\
\hline & SD & & 0.92 & 1.46 \\
\hline
\end{tabular}

indicates $p<0.05$ (uncorrected t-test probability)

$\%$ indicates the percent of patients exhibiting that trait, unless otherwise indicated 
patients had a slightly higher percentage of neutrophils, which was not statistically significant $(77.18 \%$ vs $70 \%$, NS). Appendicitis patients had significantly lower blood creatinine level $(0.78$ vs $1.54 \mathrm{mg} / \mathrm{dL}, p=0.03$ uncorrected). The two groups did not yield significantly different RNA quantities from blood, and the efficiency of RNA amplification for microarray labeling was similar.

\section{Identification of RNA biomarkers for appendicitis in whole blood}

A scatterplot of the expression patterns in the 2 groups (Fig. 1) suggested that there was excellent linearity of quantitation over roughly $7 \log 2$ orders of magnitude, with globins being the most highly and identically expressed transcripts between groups. By comparing the expression profiles of the two groups, and filtering for both a $t$-test probability $<0.05$ and a fold-change of $>2.0$, 37 transcripts were identified as significantly differentially expressed (Table 2). Hierarchical clustering of the 37 DEG was conducted to observe the pattern of covariance of the transcripts in these patients. A heatmap of the expression of these 37 transcripts across all 20 patients in the discovery set is shown in Fig. 2.

\section{Functional analysis of DEG transcripts}

Of the well annotated transcripts, several had prior published relationships to infection, immunity, or inflammation, or stress/injury: notably, alkaline phosphatase liver/bone/ kidney isoform (ALPL), carbonic anhydrase IV (CA4), chemokine (C-X-C motif) receptor 1 (CXCR1/IL-8 receptor $\alpha$ ),

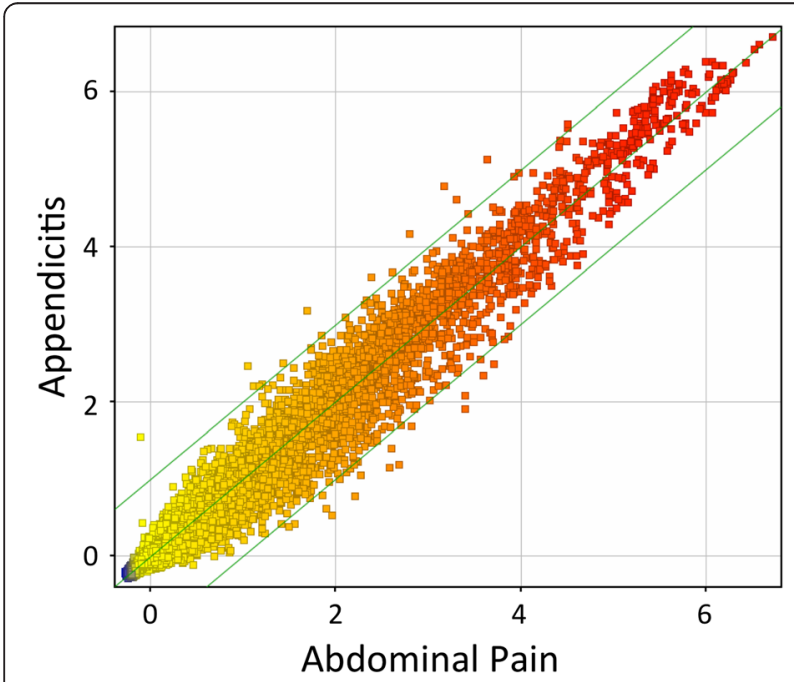

Fig. 1 Scatterplot of transcript levels in patients with appendicitis. Whole blood RNA from patients with acute, surgically confirmed appendicitis $(n=11)$ or abdominal pain $(n=9)$ was profiled for the expression level of 45,966 transcripts on Illumina BeadChip Arrays (12v4). The expression level of each transcript was averaged within groups and plotted on a log2 scale to reveal transcripts which differ between more than 2-fold between groups (outside parallel lines) defensin $\alpha 1$ (DEFA1), defensin $\alpha 3$ (DEFA3), IgG Fc receptor IIb (FCGR3B/CD16B), interleukin 8 receptor ß (CXCR2/ IL8RB), ninjurin 1, (NINJ1), prokinectin 2 (PROK2), and superoxide dismutase 2 (SOD2). In addition to their logical connection to appendicitis, which often has an infectious etiology, certain aspects of this expression pattern increase the confidence that some of these changes are non-random: 1) multiple probe sets identifying the same transcript (DEFA1), 2) 'hits' on highly related transcripts, such as DEFA1 and DEFA3, as well as CXCR1 (IL8 receptor $\alpha$ ) and CXCR2 (IL8 receptor ß).

\section{Defensins}

To understand the defensin pathway, the $5 \alpha$-defensin transcripts in the DEG list, which are all variant transcripts from the DEFA locus at 8p21.3, were averaged to create a 'defensin score', and then compared between groups (Table 1). Using a threshold determined by the mean of all 20 patients (1.87), 6 of 9 (67\%) patients with other abdominal disorders showed elevated defensins, while only 1 of 11 (9\%) of appendicitis patients had elevated defensin mRNA (see defensin cluster in Fig. 2). Surprisingly, the defensin score was essentially uncorrelated with white blood cell count (WBC) $(r=0.07)$ and neutrophil \% $(r=0.15)$.

\section{Other immune/inflammatory pathways}

Interestingly, 3 of the 37 DEG (LILRA3, CXCR1/IL8RA, FCGR3A), which were higher in appendicitis patients compared to abdominal pain patients, are near or exact matches to transcripts discovered previously as downregulated by exposure of isolated human neutrophils to $E$. Coli [18]. However, across the 20 patients, they were not inversely correlated with defensin expression (LILRA = 0.02 , CXCR1 $=-0.02$, FCGR3A $=-0.33$ ), suggesting they are regulated independently of infectious markers.

\section{Ribosomal transcripts}

While it is widely assumed that ribosomal RNAs (rRNA), such as $18 \mathrm{~S}$ and $28 \mathrm{~S}$ non-coding RNAs are 'invariant', or 'housekeeping' transcripts, there is considerable evidence that they are carefully regulated in cases such as granulocyte activation [19], and differ significantly in prostate cancer [20], and in hepatitis C infected livers [21]. In fact, early studies with PHA-activated human lymphocytes demonstrated as much as 8-fold increases in rRNA levels within $20 \mathrm{~h}[22,23]$. Furthermore, if the observed changes were due to some type of loading or processing anomaly, then we would expect all of the ribosomal RNAs to be affected in the same direction, when in fact, $18 \mathrm{~S}$ and $28 \mathrm{~S}$ noncoding transcripts were increased in appendicitis, but most of the transcripts coding for ribosomal proteins were decreased, suggesting that this is a regulated process. 
Table 2 Differentially expressed genes (DEG) sorted by functional grouping

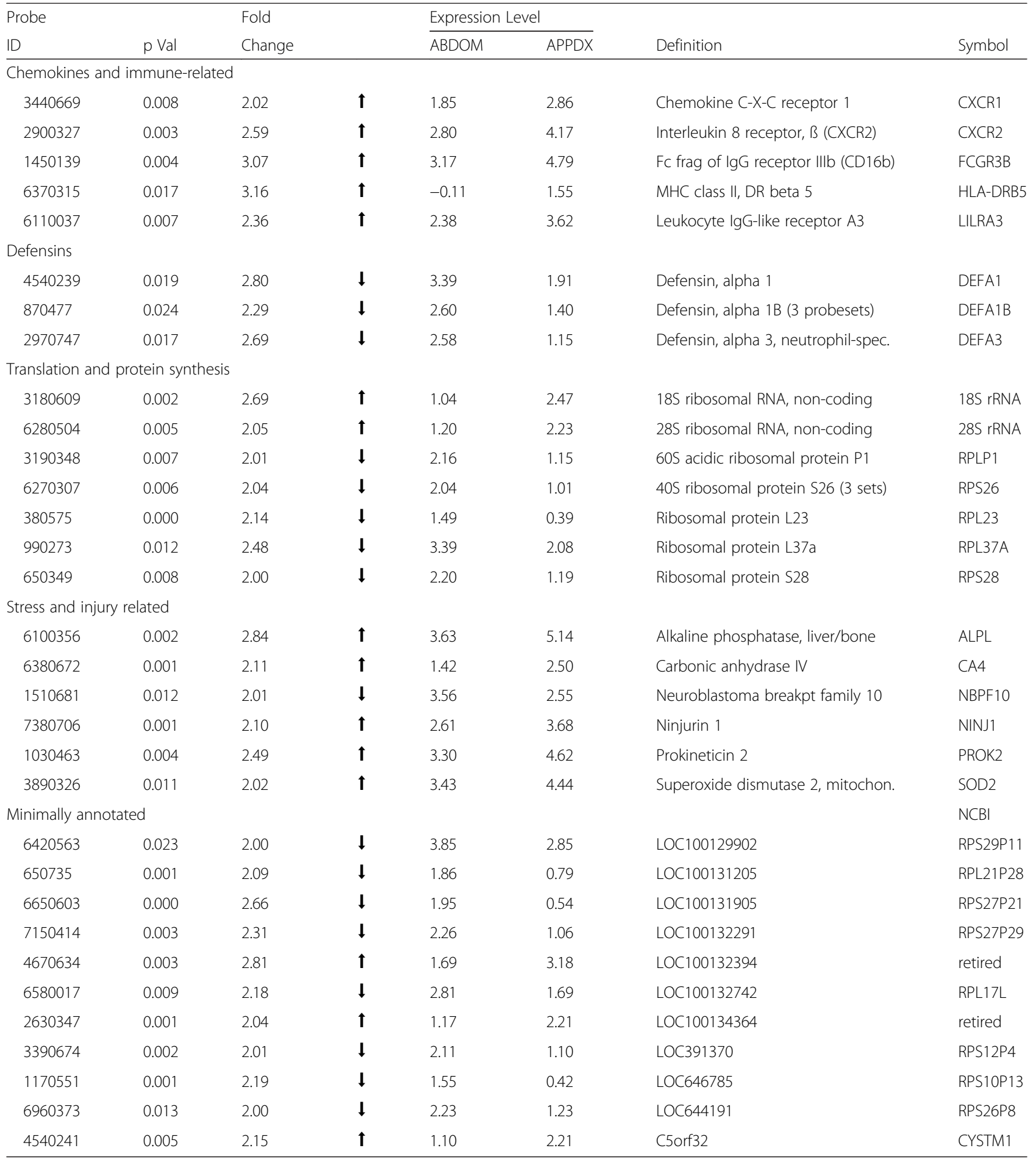

\section{Minimally annotated transcripts}

Of the 37 DEG, 11 transcripts were minimally annotated, i.e. 'predicted transcript', but further manual annotation using NCBI Gene revealed high likelihood assignments. Remarkably, 8 of the 11 transcripts were identified as ribosomal protein pseudogenes, which is quite unlikely to have occurred by chance. Two transcripts have been discontinued, and the eleventh was identified as CYSTM1 (C5ORF32), which is a cysteine-rich transmembrane module-containing protein that 2-hybrid screens identified as an inhibitor of the glucagon-like peptide 1 receptor (GLP-1R) [24]. 


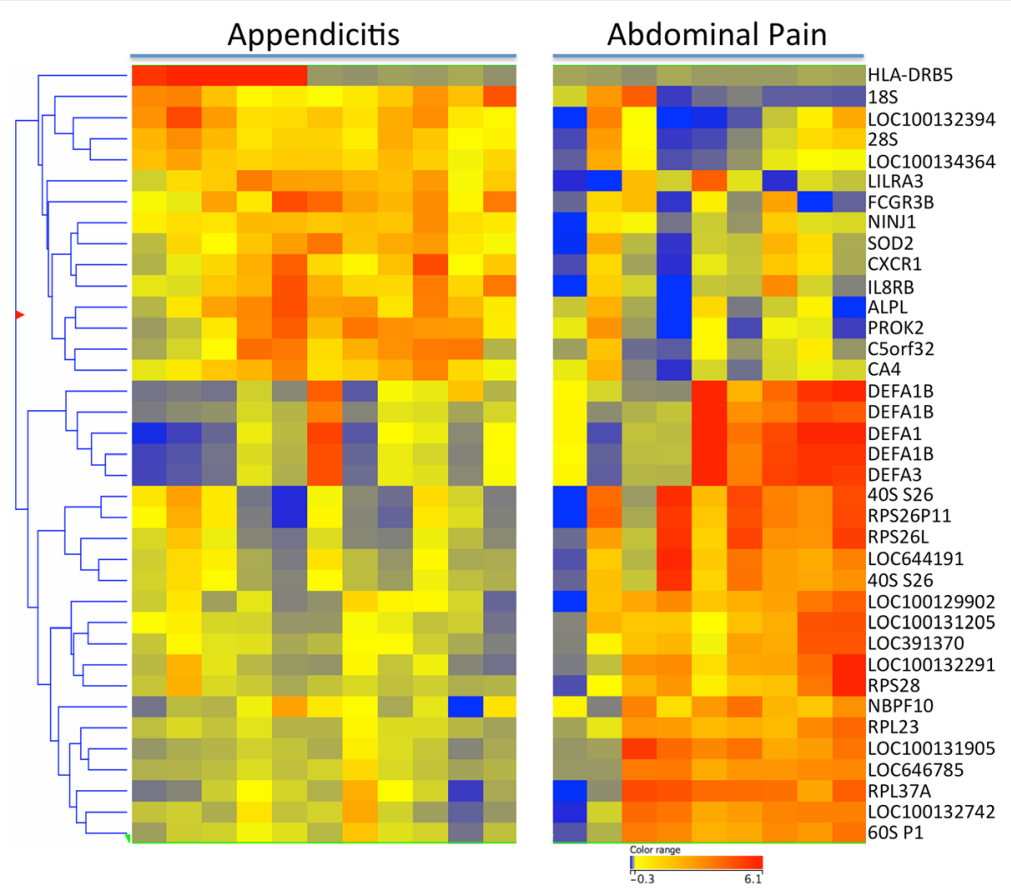

Fig. 2 Hierarchical clustering of 37 differentially expressed genes in appendicitis patients. Transcripts which differed between groups by $>2$-fold with a t-test probability of $<0.05$ (uncorrected) were identified by combined filtering. Following a per-gene normalization, DEGs were subjected to hierarchical clustering to identify patterns of covariance among the transcripts. The upper block of transcripts from HLA-DRB5 to CA4 are relatively higher in APP patients (red) compared to patients with other types of abdominal pain (yellow to blue). Conversely, transcripts from defensins (DEFA) and ribosomal transcripts, were relatively lower in APP than abdominal pain patients

\section{Prediction of appendicitis from DEG}

The PLSD model built on the 37 DEG list, was $100 \%$ accurate and specific within the discovery set, which is not surprising given the ability of PLSD models to accurately 'fit' data to outcomes. As shown in Fig. 3, the first 3 latent factors in the PLSD model demonstrate tight clustering of the appendicitis patients (blue) distinct from patients presenting with other abdominal pain (red). Clearly, 7 of 9 abdominal patients can be discriminated by only the first latent factor (t0, X-axis). Two abdominal patients, one with a GI bleed and one with diverticulitis, are poorly discriminated by the to latent factor shown in the X-axis, but are readily discriminated by one of the two other factors ( $\mathrm{Y}$ or $\mathrm{Z}$ axis). To determine whether all 37 transcripts were necessary for prediction, 16 transcripts with a loading of $>0.2$ in the PLSD model were used to rebuild a new PLSD prediction model (Additional file 4: Table S4). This smaller model, which omitted the defensins, remained quite strong, predicting $100 \%$ of abdominal cases, $90.9 \%$ of appendicitis cases, for an overall accuracy of $95 \%$.

Validation of PLSD prediction model in unrelated samples To determine the robustness of the prediction model, a separate group of patients derived from the same overall cohort were similarly processed for whole blood RNA,

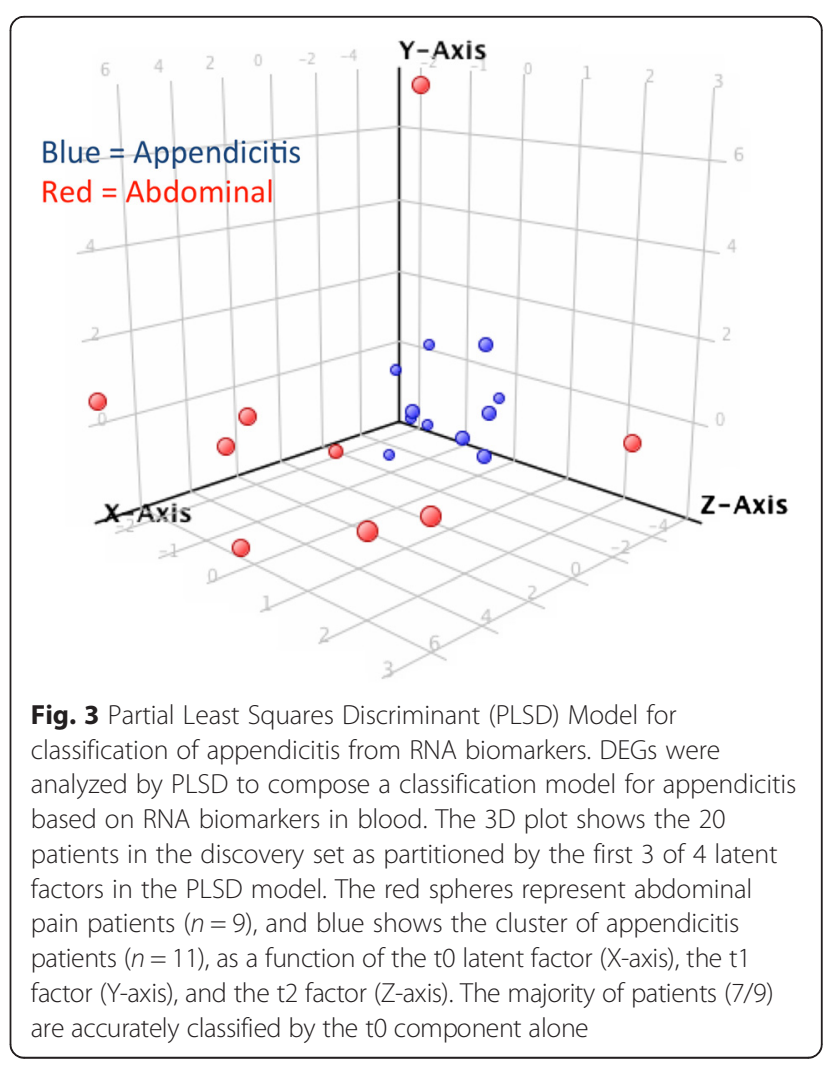


and hybridized independently to Illumina HT 12v4 Beadchip arrays. With only minimal normalization to correct for minor loading and hybridization differences, the PLSD prediction model was applied to the normalized values for the 37 transcripts in the model. The PLSD prediction model correctly identified 8 of 9 true appendicitis patients $(88.9 \%)$ and predicted 3 of 4 patients $(75 \%)$ with hernias as being 'abdominal pain'. Nearly $90 \%$ sensitivity in an unrelated cohort quantified on a different microarray run is encouraging toward the potential robustness of the model. Notably, the PLSD model includes no clinical variables, such as fever or white cell count.

\section{Behavior of the RNA biomarkers in non-appendicitis infections}

In 5 patients clinically diagnosed with LRI, which were not included in PLSD training, the model predicts 4 of 5 as appendicitis (80 \%), suggesting that the model may be sensitive to generalized infectious or inflammatory signals in blood. Using the 16 DEG model, only $60 \%$ were diagnosed as appendicitis. As shown in Fig. 4, some transcripts, such as FCGR3 and NINJ1, were relatively selectively elevated in APP, but not LRI. Other transcripts, especially defensins, were much more sensitive to LRI than APP, showing 4-5 fold elevations in LRI versus HER, and 20-fold elevations in LRI vs APP. Most transcripts, as demonstrated by CXCR2/IL8Rß, LILRA3, and ALPL, showed roughly similar changes in LRI and APP. Of the 37 transcripts, 10 are relatively selective for APP, 8 are selective for LRI, and 19 behave similarly in both APP and LRI.

\section{Validation of selected biomarkers using ddPCR in a larger cohort}

To confirm and extend the microarray-based studies, an additional 29 patients (12 APP, 17 ABD) were recruited and combined with all available samples from the prior studies to quantitate the RNA biomarkers using droplet digital PCR (ddPCR) as an independent method. As shown in Fig. 5a, the results confirm the microarray findings that appendicitis patients show significantly elevated circulating levels of mRNA for ALPL and IL8RB, while showing reduced levels of DEFA1 compared to patients with other abdominal conditions (ABD). Abdominal patients showed significantly elevated ALPL, IL8RB, and DEFA1 compared to patients with hernia (HER). Thus, APP patients are characterized as having elevated ALPL and IL8RB, without an increase in DEFA1. In contrast, patients with active lung infections showed strongly activated DEFA1 mRNA levels with only small changes in ALPL and IL8RB (Fig. 5b). The relatively high standard error in the lung group (LRI) is because 2 of the 5 patients had marginal infections, one associated with a primary diagnosis of pulmonary

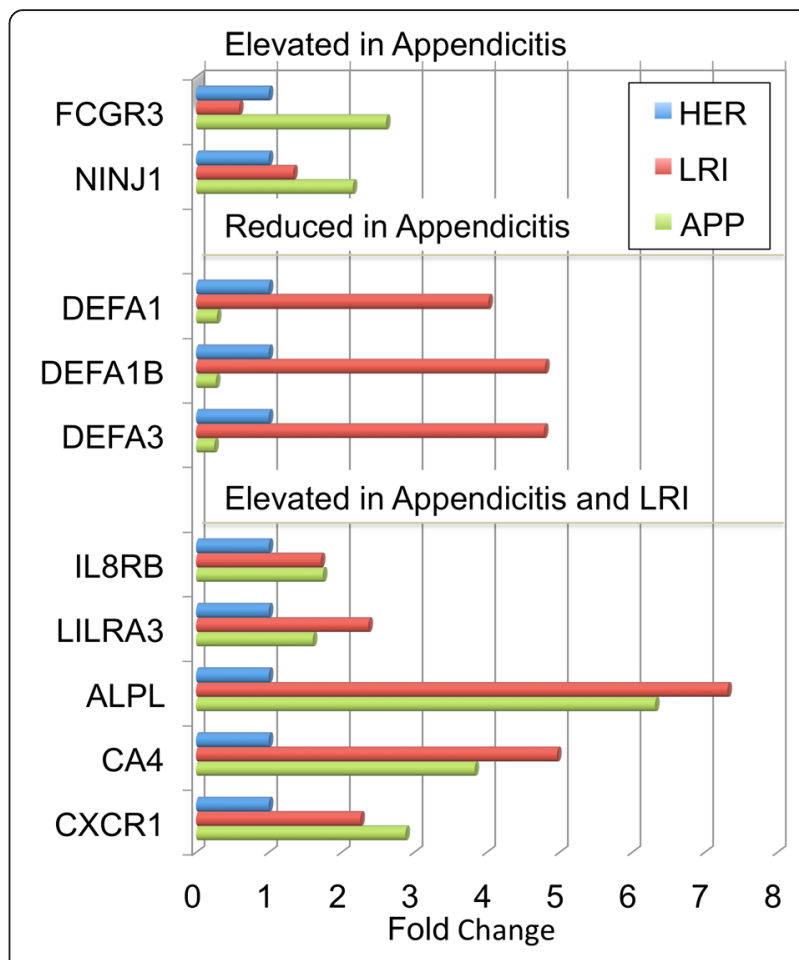

Fig. 4 Behavior of DEG biomarkers in a validation cohort. The 37 DEG biomarker set was applied to transcript expression levels in patients being treated for either appendicitis (APP, green bars), lower respiratory infection (LRI, red bars), or hernias (HER, blue bars). Representative transcripts, such as Fc gamma receptor 3 (FCGR3) and ninjurin 1 (NINJ1) are shown, in which the transcript behaves with relatively selective induction in APP, relative to HER or LRI. Conversely, transcripts in the defensin family (DEFA1, DEFA3), are significantly elevated in HER patients, relative to APP, but are strikingly induced in LRI patients. Most transcripts, such as alkaline phosphatase (ALPL) and the CXCL8 (IL-8) receptors (CXCR2/IL8RB, CXCR1), were induced in both APP and LRI patients

embolism, and the other with a mild respiratory infection, and both showed small increases in DEFA1 levels. A separate ongoing study is examining these markers in pulmonary infections, but the validity of DEFA1 mRNA is supported by recent microarray studies [25].

\section{Discussion}

Currently, there are no approved serum or urine biomarkers for appendicitis. As noted earlier, abdominal pain is one of the most common complaints in the ED, and thus blood biomarkers represent an important unmet need in clinical medicine. In this discovery and validation study, we have identified a small set of RNA transcripts associated with appendicitis. Overall, a prediction model built on these markers was able to differentiate appendicitis from other forms of intra-abdominal pathology, such as diverticulitis and hernias. Appendicitis is thought to be an inflammatory disease, similar to diverticulitis or colitis; however, there was differing activation of certain mRNA 


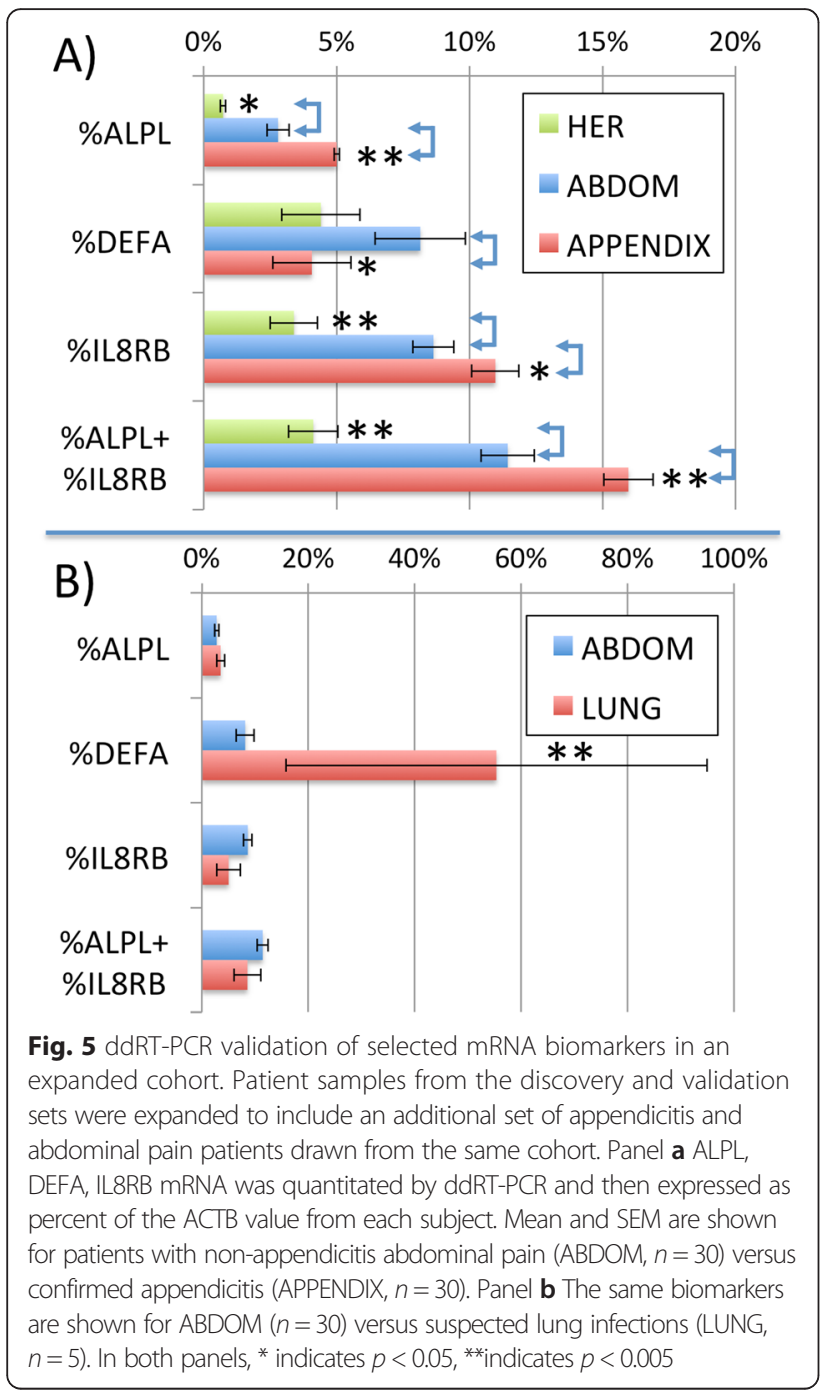

biomarkers between these conditions. Furthermore, the 37 DEG markers do not correlate with white blood cell count, per se, but a careful examination of the transcripts suggests that the RNA biomarkers may be measuring the activation state of immune cells, especially neutrophils.

The pattern of transcriptome changes in blood may help to refine our understanding of the etiology and progression of acute appendicitis, as shown schematically in Fig. 6. The classic explanation for appendicitis is that a fecalith or lymphoid hyperplasia blocks the outflow of the appendix, resulting in obstruction and ischemia [26]. Outflow obstruction may produce local changes that favor undesirable changes in the appendix microbiome. Several recent studies, including next-generation sequencing (NGS) of the $16 \mathrm{~S}$ regions of the microbiome, have suggested that relatively selective changes in Fusobacteria species are associated with appendicitis [27-30]. Fusobacteria, a type of gram-negative bacteria, can induce toxicity in adjacent host cells, and colitis-like

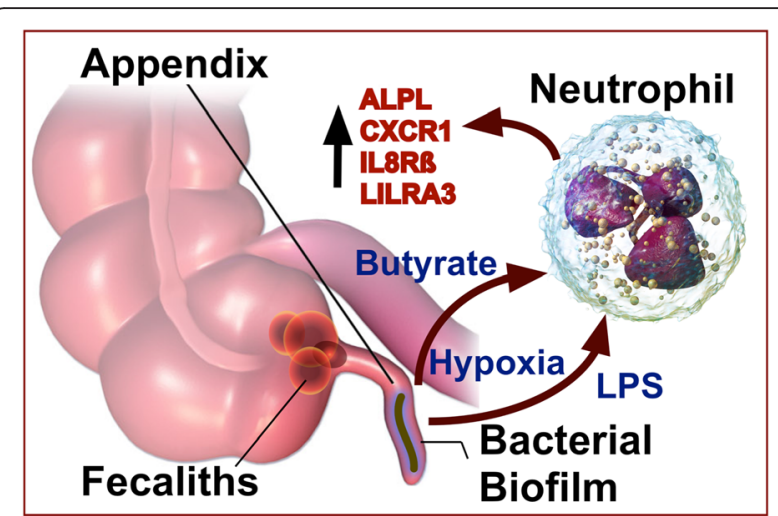

Fig. 6 Model of appendicitis biomarker pathophysiology. It is believed that compacted fecal bodies, termed fecaliths, may occlude the outflow tract of the appendix, causing inflammatory conditions that are conducive to infection in the appendix. Microbiome analysis of inflamed appendices typically indicates a predominance of biofilm-forming bacteria, such as Fusobacteria. The biofilm protects the bacteria from antibiotics, and from direct immune attack, but soluble factors produced by the bacteria, such as LPS (endotoxins) and butyrate, or cellular factors such as hypoxia and CXCL8, can diffuse into adjacent lymphatic and circulatory beds to activate neutrophils. The primed neutrophils respond with elevated transcript levels of alkaline phosphatase (ALPL), interleukin-8 receptor beta (CXCR2/IL8Rß) and related biomarkers of local infection. Background images of appendix and neutrophil courtesy of Blausen.com staff, Wikiversity Journal of Medicine

symptoms in mice, potentially by producing butyric acid (butyrate) [31].

RT-PCR analysis confirms that inflamed appendix tissue has elevated $\alpha$-defensin and CXCL8 (IL-8) mRNA levels [32]. Most studies have observed higher circulating CXCL8 levels in patients with appendicitis [33-35], and the inflamed appendix is known to locally express elevated CXCL8 [36]. Likewise, Fusobacterium nucleatum biofilms stimulate CXCL8 production in human oral epithelium cell lines [37] and Fusobacterium necrophorum induces CXCL8 production in cultured mesothelial cells [38]. Neutrophils attracted to sites of mucosal inflammation are a major component of the normal maintenance of barrier functions, and they are responsive to a variety of soluble signals within the intestinal microenvironment, including hypoxia [39].

Thus, the absence of elevated $\alpha$-defensin transcripts in the presence of elevated levels of mRNA for both CXCL8/IL-8 receptors suggests that circulating immune cells are primed by CXCL8, LPS, butyrate, and local hypoxia known to be produced in the inflamed appendix. However, it is possible that the immune cells are not directly contacting the bacterial infection, which would elevate defensins, as demonstrated clearly in the LRI patients. The increased mRNA levels for ALPL, which is a secondary granule constituent, as opposed to defensins and MPO, which are azurophilic granule components, is consistent with the pre-existing view that secondary 
granules respond to particulate and soluble neutrophil stimulators, while the azurophilic granule is principally responsive only to phagocytizable particles [40].

In addition to the CXCL8 receptors, several other transcripts appear to be plausible biomarkers of localized inflammation. Notably, ALPL, along with IL8RB/CXCR2, was identified as an expression biomarker of asthma inflammatory subtypes [41]. In addition to these interesting innate immune markers, the results revealed unexpected changes in the ribosomal system. Humans utilize 4 ribosomal RNAs, which are non-coding (5S, 5.8S, 18S, 28S), and $\sim 80$ ribosomal proteins to build multimeric translation complexes. Additionally, there are $\sim 2000$ ribosomal protein pseudogenes, which are thought to derive from inactivated duplications, but may be processed to varying degrees, and could have regulatory functions [42]. Transcripts for $18 \mathrm{~S}$ and $28 \mathrm{~S}$, both originating from multiple $45 \mathrm{~S}$ genes, were increased in the appendicitis blood RNA, which could be due to both increased transcription from active rDNA genes [43], as well engagement of previously inactive rDNA transcription units [23]. Conversely, most of the coding transcripts, such as RPLP1 and RPS26, were decreased in the blood of appendicitis patients. Because the specific pattern of ribosomal proteins defines the type of RNAs that are engaged and translated [44], it is possible that the translational machinery is being re-geared to react to pathogens. Unexpectedly, most of the poorly annotated transcripts were mapped to ribosomal protein pseudogenes, suggesting that either the probesets are incorrectly detecting a change in coding ribosomal protein transcripts, or the pseudogenes are somehow regulated in conjunction with the reconfigured translational machinery. Nonetheless, the ribosomal transcript changes reflect a small fraction of the ribosomal machinery, not a sweeping change in 'housekeeping' genes. Conceptually, the pattern of chemokine, defensin, stress-related, and ribosomal processing changes is consistent with the immune system being 'primed' as the immune cells pass through an inflammatory field created by a localized biofilm infection.

Other transcripts were readily associated with tissue injury or inflammation, but not previously associated with pathogen infection (Table 2). For instance, NINJ1 was identified as a transcript strongly upregulated after peripheral nerve injury [45]. PROK2 is elevated in colitis tissue [46], which, like appendicitis, is an inflammatory condition in the GI tract. Likewise, ALPL has a wellknown role in modulating diverse inflammatory conditions not limited to infectious disease [47]. Consistent with the current data, human neutrophils treated ex vivo with TNF- $\alpha$ and/or GM-CSF also show induction of transcripts for CXCR1, CXCR2/IL8RB, FCGR3B, NINJ1, and PROK2 [48].

Other investigators have sought to develop protein biomarkers for appendicitis in the blood, such as bilirubin
[49], C-reactive protein (CRP) [50], and pro-calcitonin (PCT) [51]. However, recent comparisons of these biomarkers had difficulty improving on a purely clinical prediction model, such as the Alvarado score $(\mathrm{ROC}=0.74$, vs $\mathrm{CRP}=0.61, \mathrm{PCT}=0.69$ ) [52]. Recently, a combination of WBC, CRP, and MRP8/14 (S100A8/S100A9) was shown to be $96 \%$ sensitive, but $43 \%$ specific for acute appendicitis [50]. Likewise, a multivariate model built on plasma protein levels of serum amyloid (SAA), myeloperoxidase (MPO), and MMP9 was less diagnostic than a largely clinical model ( $\mathrm{ROC}=0.71$ vs 0.91 clinical model) [53].

While RNA-based diagnostic tests are currently on the market for breast cancer progression (MammaPrint, OncoType Dx), transplant rejection (AlloMap), and coronary artery disease (CorusCAD), to our knowledge, this is the first report to assess blood RNA as a potential biomarker of appendicitis. Among the strengths of the present approach is that the test and validation sets included controls for surgical, inflammatory, and infectious factors. Further, the RNA profiling was broad and largely unbiased, and detected the same key pathways in the test and validation study. Third, unlike protein biomarkers, which can be difficult to measure due to interference by other high-abundance proteins, mRNA is highly reproducible, very sensitive to perturbation, and can be localized to specific cell types (e.g. neutrophils) for disease monitoring.

In addition to these strengths, the present study has certain limitations. First, the sizes of the cohorts used in the discovery phase were modest. As a consequence, the range of intra-abdominal diseases that we were able to assess was limited. The LRI biomarkers must be assessed cautiously due to the small and heterogeneous group. Second, due to randomness in enrollment, the ethnicity was unbalanced between groups, and this may have introduced a bias into the interpretation. However, analysis of the ddPCR data by race and gender did not identify differences (not shown). Nonetheless, we have successfully utilized a genome-wide RNA transcript profiling to identity potential genomic biomarkers of appendicitis. Overall, the detected biomarkers are consistent with prior published evidence that bacterial biofilms in the appendix may be an important putative pathophysiological mechanism in appendicitis that can be detected by the RNA profile of circulating neutrophils.

\section{Conclusions}

- Transcript profiling of blood identified RNA biomarkers in patients with confirmed appendicitis.

- Secondary granule markers, such as ALPL were increased, while azurophilic granule markers, such as DEFA1, decreased in appendicitis.

- Lung infections increased azurophilic markers more than secondary granule markers. 
- Transcript changes associated with acute appendicitis are consistent with immune priming by a bacterial biofilm, probably transmitted by soluble factors such CXCL8, LPS, and local hypoxic conditions that activate neutrophils transiting the inflamed area.

\section{Additional files}

Additional file 1: Table S1. Inclusion and Exclusion Criteria. Describes the inclusion criteria for the patients enrolled in the prospective, observational study of biomarkers for appendicitis. (DOCX 55 kb)

Additional file 2: Table S2. Clinical Information on all Patients Analyzed. Describes the age, gender, race, body mass index (BMI), smoking, white blood cell count (WBC), pathology of the appendix, computed tomography $(\mathrm{CT})$ results, and final diagnosis of the patients included in the final analysis. (XLSX $16 \mathrm{~kb}$ )

Additional file 3: Table S3. Appendix List of Primers. (XLSX 54 kb) Additional file 4: Table S4. Sixteen gene list highly weighted in 37 gene PLS model. Describes the 16 gene transcripts that were the most highly weighted from the larger 37 gene lists. These 16 transcripts were used to build a second PLS prediction model as described. (DOCX $72 \mathrm{~kb}$ )

\section{Acknowledgements}

The authors are grateful to the ongoing generosity of the Abramson Family Trust for financial support of the project. AN is a recipient of a Gill Fellowship.

\section{Availability of data and materials}

The raw Illumina data files from each subject have been deposited in the NCB Gene Expression Omnibus, and are available at http://www.ncbi.nlm.nih.gov/ geo/query/acc.cgi?acc=GSE83091 under the accession numbers GSE83091.

\section{Authors' contributions}

LC and TM conceived and designed the studies, and wrote the manuscript with input from all authors. $L C, D D, K V, J L, M S$, and RL identified and consented patients, and collected clinical and laboratory data. IT, AN, and TM conducted RNA isolations, microarray analysis, and statistical analysis. All authors have read and approved the manuscript.

\section{Competing interests}

LC and TM have relevant intellectual property in the area of blood markers of human disease, including appendicitis, lower respiratory infection, and cardiovascular diseases. The other authors do not report any competing interests.

\section{Consent for publication}

No personally identifiable information is disclosed and the participants have agreed to publication of the data in unidentified, aggregate form.

\section{Ethics approval and consent to participate}

All subjects provided informed consent to participate in this research protocol, which was reviewed and approved by The George Washington University Institutional Review Board (IRB\#040704).

\section{Author details}

'Department of Anesthesiology and Critical Care Medicine, The George Washington University Medical Center, 2300 I Street, NW Ross 443, Washington, DC 20037, USA. ²Department of Medicine, Division of Genomic Medicine, The George Washington University Medical Center, 2300 I Street, NW Ross 443, Washington, DC 20037, USA. ${ }^{3}$ Department of Surgery, The George Washington University Medical Center, 2300 I Street, NW Ross 443, Washington, DC 20037, USA. ${ }^{4}$ Department of Microbiology, Immunology, and Tropical Medicine, The George Washington University Medical Center, 2300 I Street, NW Ross 443, Washington, DC 20037, USA. ${ }^{5}$ Department of Emergency Medicine, The George Washington University Medical School and GW Medical Faculty Associates, Washington, DC, USA. ${ }^{6}$ The Department of Medicine, Veterans Affairs Medical Center, The George Washington University Medical Center, 2300 I Street, NW Ross 443, Washington, DC 20037, USA.
Received: 7 March 2016 Accepted: 9 June 2016

Published online: 15 July 2016

\section{References}

1. Bhuiya F, Pitts S, McCaig L. Emergency Department visits for chest pain and abdominal pain: United States, 1999-2008. CDC, NCHS Data Brief. 2010:43:1-8.

2. Charfi S, Sellami A, Affes A, Yaich K, Mzali R, Boudawara TS. Histopathological findings in appendectomy specimens: a study of 24,697 cases. Int J Colorectal Dis. 2014;29(8):1009-12.

3. Drake FT, Florence MG, Johnson MG, Jurkovich GJ, Kwon S, Schmidt Z, Thirlby RC, Flum DR, Collaborative S. Progress in the diagnosis of appendicitis: a report from Washington State's Surgical Care and Outcomes Assessment Program. Ann Surg. 2012;256(4):586-94.

4. Seetahal SA, Bolorunduro OB, Sookdeo TC, Oyetunji TA, Greene WR, Frederick W, Cornwell lii EE, Chang DC, Siram SM. Negative appendectomy: a 10-year review of a nationally representative sample. Am J Surg. 2011;201(4):433-7.

5. Kirkil C, Karabulut K, Aygen E, Ilhan YS, Yur M, Binnetoglu K, Bulbuller N. Appendicitis scores may be useful in reducing the costs of treatment for right lower quadrant pain. Ulus Travma Acil Cerrahi Derg. 2013;19(1):13-9.

6. Memon ZA, Irfan S, Fatima K, lqbal MS, Sami W. Acute appendicitis: diagnostic accuracy of Alvarado scoring system. Asian journal of surgery/ Asian Surgical Association. 2013;36(4):144-9.

7. Teixeira PG, Demetriades D. Appendicitis: changing perspectives. Adv Surg. 2013:47(1):119-40.

8. Poortman P, Oostvogel HJM, Bosma E, Lohle PNM, Cuesta MA, de Lange-de Klerk ESM, Hamming JF. Improving Diagnosis of Acute Appendicitis: Results of a Diagnostic Pathway with Standard Use of Ultrasonography Followed by Selective Use of CT. J Am Coll Surg. 2009;208(3):434-41.

9. Collins GB, Tan TJ, Gifford J, Tan A. The accuracy of pre-appendectomy computed tomography with histopathological correlation: a clinical audit, case discussion and evaluation of the literature. Emerg Radiol. 2014;21(6):589-95.

10. Rosen MP, Ding A, Blake MA, Baker ME, Cash BD, Fidler JL, Grant TH, Greene FL, Jones B, Katz DS, et al. ACR Appropriateness Criteria ${ }^{\oplus}$ Right Lower Quadrant Pain-Suspected Appendicitis. J Am Coll Radiol. 2011;8(11):749-55.

11. Ahn S, group L. LOCAT (low-dose computed tomography for appendicitis trial) comparing clinical outcomes following low-vs standard-dose computed tomography as the first-line imaging test in adolescents and young adults with suspected acute appendicitis: study protocol for a randomized controlled trial. Trials. 2014;15:28.

12. Miglioretti $\mathrm{DL}$, Johnson E, Williams A, Greenlee RT, Weinmann S, Solberg LI, Feigelson HS, Roblin D, Flynn MJ, Vanneman N, et al. The use of computed tomography in pediatrics and the associated radiation exposure and estimated cancer risk. JAMA Pediatr. 2013;167(8):700-7

13. Wai S, Ma L, Kim E, Adekunle-Ojo A. The utility of the emergency department observation unit for children with abdominal pain. Pediatr Emerg Care. 2013;29(5):574-8.

14. Seo H, Lee KH, Kim HJ, Kim K, Kang S-B, Kim SY, Kim YH. Diagnosis of Acute Appendicitis With Sliding Slab Ray-Sum Interpretation of Low-Dose Unenhanced CT and Standard-Dose IV Contrast-Enhanced CT Scans. Am J Roentgenol. 2009:193(1):96-105.

15. Kim SY, Lee KH, Kim K, Kim TY, Lee HS, Hwang S-S, Song KJ, Kang HS, Kim YH, Rhee JE. Acute Appendicitis in Young Adults: Low- versus StandardRadiation-Dose Contrast-enhanced Abdominal CT for Diagnosis. Radiology. 2011;260(2):437-45.

16. Keyzer C, Tack D, de Maertelaer V, Bohy P, Gevenois PA, Van Gansbeke D. Acute Appendicitis: Comparison of Low-Dose and Standard-Dose Unenhanced Multi-Detector Row CT. Radiology. 2004;232(1):164-72.

17. da Huang W, Sherman BT, Lempicki RA. Systematic and integrative analysis of large gene lists using DAVID bioinformatics resources. Nat Protoc. 2009: 4(1):44-57.

18. Subrahmanyam $\mathrm{V}$, Yamaga $S$, Prashar $Y$, Lee HH, Hoe NP, Kluger $Y$, Gerstein M, Goguen JD, Newburger PE, Weissman SM. RNA expression patterns change dramatically in human neutrophils exposed to bacteria. Blood. 2001; 97(8):2457-68.

19. Poortinga G, Wall M, Sanij E, Siwicki K, Ellul J, Brown D, Holloway TP, Hannan RD, McArthur GA. c-MYC coordinately regulates ribosomal gene chromatin remodeling and Pol I availability during granulocyte differentiation. Nucleic Acids Res. 2011;39(8):3267-81.

20. Uemura M, Zheng Q, Koh CM, Nelson WG, Yegnasubramanian S, De Marzo AM. Overexpression of ribosomal RNA in prostate cancer is common but not linked to rDNA promoter hypomethylation. Oncogene. 2012;31(10):1254-63. 
21. Congiu M, Slavin JL, Desmond PV. Expression of common housekeeping genes is affected by disease in human hepatitis C virus-infected liver. Liver Int. 2011;31(3):386-90.

22. Derenzini M, Farabegoli F, Pession A, Novello F. Spatial redistribution of ribosomal chromatin in the fibrillar centres of human circulating lymphocytes after stimulation of transcription. Exp Cell Res. 1987;170(1):31-41.

23. Haaf T, Hayman DL, Schmid M. Quantitative determination of rDNA transcription units in vertebrate cells. Exp Cell Res. 1991;193(1):78-86.

24. Huang X, Dai FF, Gaisano G, Giglou K, Han J, Zhang M, Kittanakom S, Wong V Wei $L$, Showalter AD, et al. The identification of novel proteins that interact with the GLP-1 receptor and restrain its activity. Mol Endocrinol. 2013;27(9): 1550-63.

25. Tsalik EL, Henao R, Nichols M, Burke T, Ko ER, McClain MT, Hudson LL, Mazur A Freeman DH, Veldman T, et al. Host gene expression classifiers diagnose acute respiratory illness etiology. Sci Transl Med. 2016:8(322):322ra311.

26. Ramdass MJ, Young Sing Q, Milne D, Mooteeram J, Barrow S. Association between the appendix and the fecalith in adults. Canadian journal of surgery Journal canadien de chirurgie. 2015;58(1):10-4.

27. Jackson HT, Mongodin EF, Davenport KP, Fraser CM, Sandler AD, Zeichner SL. Culture-independent evaluation of the appendix and rectum microbiomes in children with and without appendicitis. PLoS One. 2014:9(4):e95414.

28. Zhong D, Brower-Sinning R, Firek B, Morowitz MJ. Acute appendicitis in children is associated with an abundance of bacteria from the phylum Fusobacteria. J Pediatr Surg. 2014;49(3):441-6.

29. Guinane CM, Tadrous A, Fouhy F, Ryan CA, Dempsey EM, Murphy B, Andrews E, Cotter PD, Stanton C, Ross RP: Microbial composition of human appendices from patients following appendectomy. mBio 2013;4(1). doi: 10.1128/mBio.00366-12

30. Swidsinski A, Dorffel Y, Loening-Baucke $V$, Theissig F, Ruckert JC, Ismail M, Rau WA, Gaschler D, Weizenegger M, Kuhn S, et al. Acute appendicitis is characterised by local invasion with Fusobacterium nucleatum/ necrophorum. Gut. 2011;60(1):34-40.

31. Ohkusa T, Okayasu I, Ogihara T, Morita K, Ogawa M, Sato N. Induction of experimental ulcerative colitis by Fusobacterium varium isolated from colonic mucosa of patients with ulcerative colitis. Gut. 2003;52(1):79-83.

32. Arlt A, Bharti $R$, Ilves I, Hasler R, Miettinen P, Paajanen H, Brunke G, Ellrichmann M, Rehman A, Hauser $\mathrm{C}$, et al. Characteristic changes in microbial community composition and expression of innate immune genes in acute appendicitis. Innate Immun. 2013.

33. Kharbanda AB, Cosme Y, Liu K, Spitalnik SL, Dayan PS. Discriminative accuracy of novel and traditional biomarkers in children with suspected appendicitis adjusted for duration of abdominal pain. Acad Emerg Med. 2011;18(6):567-74.

34. Yoon DY, Chu J, Chandler C, Hiyama S, Thompson JE, Hines OJ. Human cytokine levels in nonperforated versus perforated appendicitis: molecular serum markers for extent of disease? Am Surg. 2002;68(12):1033-7.

35. Ruber M, Andersson M, Petersson BF, Olaison G, Andersson RE, Ekerfelt C. Systemic Th17-like cytokine pattern in gangrenous appendicitis but not in phlegmonous appendicitis. Surgery. 2010;147(3):366-72.

36. Murphy CG, Glickman JN, Tomczak K, Wang YY, Beggs AH, Shannon MW, Horwitz $\mathrm{BH}$. Acute appendicitis is characterized by a uniform and highly selective pattern of inflammatory gene expression. Mucosal Immunol. 2008;1(4):297-308.

37. Peyyala R, Kirakodu SS, Novak KF, Ebersole JL. Oral microbial biofilm stimulation of epithelial cell responses. Cytokine. 2012;58(1):65-72.

38. Zeillemaker AM, van Papendrecht AA H, Hart MH, Roos D, Verbrugh HA, Leguit $P$. Peritoneal interleukin-8 in acute appendicitis. J Surg Res. 1996;62(2):273-7.

39. Colgan SP. Neutrophils and inflammatory resolution in the mucosa. Semin Immunol. 2015;27(3):177-83.

40. Boxer LA, Smolen JE. Neutrophil granule constituents and their release in health and disease. Hematol Oncol Clin North Am. 1988;2(1):101-34.

41. Baines KJ, Simpson JL, Wood L, Scott R, Fibbens N, DipPathTech, Powell HJ, Cowan D, Taylor R, Cowan JO, et al. Sputum gene expression signature of 6 biomarkers discriminates asthma inflammatory phenotypes. J Allergy Clin Immunol. 2014;133:997-1007

42. Zhang Z, Harrison P, Gerstein M. Identification and analysis of over 2000 ribosomal protein pseudogenes in the human genome. Genome Res. 2002; 12(10):1466-82.

43. Shiue CN, Nematollahi-Mahani A, Wright AP. Myc-induced anchorage of the rDNA IGS region to nucleolar matrix modulates growth-stimulated changes in higher-order rDNA architecture. Nucleic Acids Res. 2014;42(9):5505-17.

44. Remacha M, Jimenez-Diaz A, Bermejo B, Rodriguez-Gabriel MA, Guarinos E, Ballesta JP. Ribosomal acidic phosphoproteins P1 and P2 are not required for cell viability but regulate the pattern of protein expression in Saccharomyces cerevisiae. MolCellBiol. 1995;15(9):4754-62.

45. Kubo T, Yamashita T, Yamaguchi A, Hosokawa K, Tohyama M. Analysis of genes induced in peripheral nerve after axotomy using cDNA microarrays. J Neurochem. 2002;82(5):1129-36.

46. Watson RP, Lilley E, Panesar M, Bhalay G, Langridge S, Tian SS, McClenaghan C, Ropenga A, Zeng F, Nash MS. Increased prokineticin 2 expression in gut inflammation: role in visceral pain and intestinal ion transport. Neurogastroenterol Motil. 2012;24(1):65-75. e12.

47. Pike AF, Kramer NI, Blaauboer BJ, Seinen W, Brands R. A novel hypothesis for an alkaline phosphatase 'rescue' mechanism in the hepatic acute phase immune response. BiochimBiophysActa. 2013;1832(12):2044-56.

48. Wright HL, Thomas HB, Moots RJ, Edwards SW. RNA-seq reveals activation of both common and cytokine-specific pathways following neutrophil priming. PLoS One. 2013:8(3), e58598.

49. D'Souza N, Karim D, Sunthareswaran R. Bilirubin; a diagnostic marker for appendicitis. Int J Surg. 2013;11(10):1114-7.

50. Huckins DS, Simon HK, Copeland K, Spiro DM, Gogain J, Wandell M. A novel biomarker panel to rule out acute appendicitis in pediatric patients with abdominal pain. Am J Emerg Med. 2013;31(9):1368-75.

51. Kaya B, Sana B, Eris C, Karabulut K, Bat O, Kutanis R. The diagnostic value of D-dimer, procalcitonin and CRP in acute appendicitis. Int J Med Sci. 2012; 9(10):909-15.

52. Wu JY, Chen HC, Lee SH, Chan RC, Lee CC, Chang SS. Diagnostic role of procalcitonin in patients with suspected appendicitis. World J Surg. 2012; 36(8):1744-9.

53. Andersson M, Ruber $M$, Ekerfelt $C$, Hallgren $H B$, Olaison $G$, Andersson RE. Can new inflammatory markers improve the diagnosis of acute appendicitis? World J Surg. 2014;38(11):2777-83.

\section{Submit your next manuscript to BioMed Central and we will help you at every step:}

- We accept pre-submission inquiries

- Our selector tool helps you to find the most relevant journal

- We provide round the clock customer support

- Convenient online submission

- Thorough peer review

- Inclusion in PubMed and all major indexing services

- Maximum visibility for your research

Submit your manuscript at www.biomedcentral.com/submit
) Biomed Central 